\title{
Artículo
}

\section{Procesos educativos no formales e itinerantes en la atención de las infancias ${ }^{*}$}

\section{Non-formal and itinerant educational processes in the care of children}

Mary Luz Acero ${ }^{* *}$

Alba M Castro Nemocón ${ }^{* *}$

Nubia Marcela Gómez ${ }^{* * *}$

William A Prieto Galindo ${ }^{* * * * *}$

\section{Resumen}

Aula Móvil es un sitio de práctica profesional itinerante en el que los docentes en formación de la Licenciatura en Educación Infantil realizan actividades lúdico-pedagógicas a las infancias y sus familias de Soacha. Objetivo. El objetivo es caracterizar los elementos constitutivos de las propuestas educativas itinerantes a ser

\footnotetext{
* Artículo original derivado del Proyecto "Procesos educativos" Financiado por Corporación Universitaria Minuto de Dios entre agosto 2018 y mayo 2019.

** Docente universitario, Corporación Universitaria Minuto de Dios. Bogota, Colombia. $\quad$ E-mail: macero@uniminuto.edu.co._ ORCID: http://orcid.org/0000-0002-3602-2784.

*** Docente universitario, Corporación Universitaria Minuto de Dios. Bogota, Colombia. E-mail: alba.castro@uniminuto.edu.co._ORCID: http://orcid.org/0000-0001-6312-3583.

**** Docente universitario, Corporación Universitaria Minuto de Dios. Bogota, Colombia. E-mail: nubiam.gomez@uniminuto.edu. ORCID: http://orcid.org/0000-0002-0714-3114.

***** Docente universitario, Corporación Universitaria Minuto de Dios. Bogota, Colombia. E-mail: william.prieto@uniminuto.edu ORCID: http://orcid.org/0000-0001-9988-8238
}

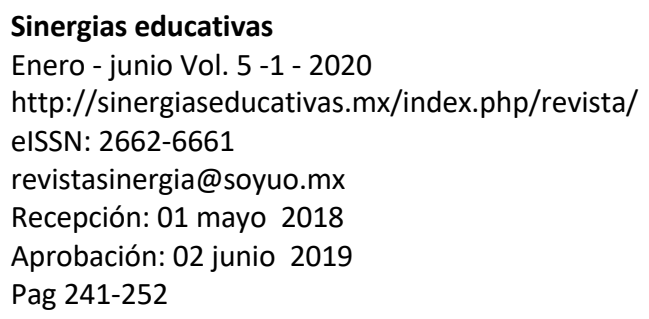

Disponible en

http://www.redalyc.org/articulo.oa?id=57356143200 18

Atribución/Reconocimiento-NoComercialCompartirlgual 4.0 Licencia Pública Internacional CC BY-NC-SA 4.0 
implementadas por los docentes en formación como acciones sociales responsables. Materiales y métodos. Se aplica una investigación de enfoque cualitativo y diseño descriptivo que muestran la relevancia de la itinerancia en la atención de las infancias vulnerables del municipio. Resultados. Los resultados permitieron evidenciar la itinerancia como una estrategia educativa no formal valiosa en la atención a población en condición de vulnerabilidad socio-económica y de difícil acceso; la educación itinerante es una alternativa de atención no formal para las infancias que viven en lugares apartados y no cuentan con cobertura. Conclusión. Este proceso educativo itinerante complementa la educación regular y ofrece aprendizajes significativos para la vida.

Palabras clave: aula móvil, itinerante, educación no formal, ambientes de aprendizaje.

\begin{abstract}
Mobile Classroom is an itinerant professional practice site in which teachers in training for the Bachelor's Degree in Early Childhood Education carry out recreational and educational activities for children and their families in Soacha. Objective. The objective is to characterize the constituent elements of the itinerant educational proposals to be implemented by teachers in training as responsible social actions. Materials and methods. An investigation of qualitative approach and descriptive design that shows the relevance of roaming in the care of vulnerable childhoods in the municipality is applied. Results. It was shown that roaming as a valuable nonformal educational strategy in the care of the population in a condition of socio-economic vulnerability and difficult access; itinerant education is an alternative of non-formal care for children living in remote areas and not covered.
\end{abstract}

Key words: mobile classroom, itinerant, non-formal education, learning environments.

\title{
Introducción
}

La educación no formal as Aquila es aquella que busca desarrollar competencias intelectuales y morales útiles para la vida. Se ofrece 
con el objeto de mejorar las capacidades humanas, ya que trabaja en potenciar competencias, valores, habilidades artísticas, recreativas y técnicas. De igual manera, persigue la protección de los recursos naturales y la participación ciudadana. Complementa la educación regular y no está sujeta al sistema de niveles y grados. Ministerio de Educación Nacional (MEN, 1994).

La educación itinerante es aquella que lleva actividades pedagógicas y lúdicas a diferentes lugares como parques, espacios verdes, salones comunales, apartados y de difícil acceso.

Los aspectos anteriores, educación no formal e itinerante, están enmarcados en la responsabilidad social que tiene la educación superior de trabajar por las problemáticas que se presentan en las diferentes comunidades. Donde es necesario trascender de la formación conceptual, disciplinar profesional y formar individuos sensibles, conscientes de cuáles son las dinámicas y situaciones que afrontan las sociedades en las que se conviven, resaltando su compromiso con la transformación, diseñando proyectos que los beneficien,

...se podrá verificar en la realidad el proceso de la formación universitaria socialmente responsable, preguntándonos si “el auténtico criterio para evaluar las universidades no es lo que nuestros estudiantes hagan, sino lo que acaben siendo y la responsabilidad con la cual trabajen en favor de sus prójimos y del mundo (Domínguez, 2012, p:16).

Este artículo muestra los resultados del segundo objetivo del proyecto de Investigación Pedagogía al Contexto Aula Móvil: Caracterizar los elementos constitutivos de las propuestas educativas implementadas en Aula Móvil como acciones sociales responsables para atender a niñas, niños, padres y cuidadores con actividades lúdico-pedagógicas en las diferentes comunas y veredas del Municipio de Soacha, sitios con vulnerabilidad socio-económica y de difícil acceso. Estas actividades están organizadas por ambientes de aprendizajes que buscaban ejercitar las dimensiones del ser de las infancias a través del juego y la recreación. 
Las aulas itinerantes se han implementado en varios sitios del mundo, con el fin de atender a la población infantil que vive lugares apartados de las ciudades o en las periferias de éstas, vulnerables en su situación económica y social. La itinerancia permite la atención e intervención de varias formas, como por ejemplo el Servicio de Apoyo Educativo Domiciliario y Aulas Hospitalarias-domiciliarias dirigidos a estudiantes en condiciones desfavorables por su condición de salud, situación geográfica, social o económica que no se les facilita recibir la atención educativa; estas iniciativas buscan la equidad y atención igualitaria para todos. A continuación, se mostrarán algunos ejemplos de estas aulas itinerantes en el mundo.

La itinerancia permite la atención inclusiva a poblaciones apartadas y de difícil acceso, tal como lo plantean Torres (2016), el MEN (2004), la UNAD (2018) y Bernal (2012) con propuestas innovadoras como el teatro y el maestro itinerante a poblaciones donde no llega la educación formal y no formal por falta de cobertura, inversión del gobierno o carencia de programas que atiendan el desarrollo integral de las infancias y juventudes.

Torres (2016), muestra las Aulas itinerantes en el circo, ofrecida a los niños y niñas cuyas familias trabajan en empresas circenses en España como medida de atención inclusiva debido a que los padres, o incluso los mismos niños se dedican al espectáculo y no pueden acudir a la escuela por los continuos desplazamientos. Para esto, nombran a un docente que vive en el circo y realiza el trabajo pedagógico con ellos de forma regular, con los parámetros de la escuela conocida con materiales didácticos y juegos.

El MEN (2004) y la UNAD (2018) ofrecen una propuesta inclusiva el Maestro Itinerante en zonas apartadas con dificultades de desplazamiento. El primero, a niños en edad preescolar y que no tienen acceso a la educación, entonces es el maestro el que se desplaza pues los niños no pueden hacerlo, éste les enseña mediante el juego. En el 2003, lograron atender a 64 escuelas con una población de 1.413 niños y niñas.

El segundo, dirigida a jóvenes y adultos que vivieron el rigor de la guerra, permite que ellos puedan retomar o iniciar sus estudios sin interferir con su cotidianidad. Las actividades académicas se 
convierten en un encuentro de saberes, acompañados por sus familiares y teniendo en cuenta los conocimientos previos de los estudiantes. Utilizan una cartilla y herramientas web 2.0 (Skype, correo electrónico, llamadas telefónicas, envío de las lecciones y videos informativos vía WhatsApp). Los encuentros se realizan en diferentes partes como sus casas, cafeterías, plaza de mercado en un ambiente agradable y significativo para adquirir los aprendizajes.

Bernal (2012), muestra una estrategia itinerante inclusiva, Radio Sutatenza, que formó a los campesinos colombianos entre 1947 y 1994; pionera en el uso de la multimedia en Latinoamérica. Se apoyaba en cartillas diseñadas para desarrollar el componente pedagógico, conocimiento del alfabeto, del cálculo matemático básico, nociones de salud individual y familiar, aprendizaje de técnicas de producción agropecuaria para mejorar la economía campesina, así como el desarrollo de valores, comportamientos cívicos y religiosos.

Covarrubias (2016) y Rodríguez-Gutiérrez (2017) mediante sus propuestas itinerantes, concebidas como estrategias de aprendizaje, trabajan por el cuidado del medio ambiente y ejercitan aspectos cognitivos, artísticos, emocionales, lúdicos y estéticos en los participantes. El primero, mediante el Microteatro que implementa la técnica de títeres Bunraku (títeres de dos tercios de la figura humana, originarios de Japón y manejados por dos o tres personas) con funciones breves trabajan por el cuidado del medio ambiente en colonias y escuelas de educación básica ubicadas contextos de vulnerabilidad social en México.

El segundo, mediante la universidad itinerante de la Mar en España, ofrece formación a los jóvenes para concientizarlos del cuidado y la importancia del mar en el desarrollo de las sociedades actuales. Busca una formación complementaria para ampliar la conciencia marítima y las capacidades personales para el trabajo en equipo.

La Fundación, Nuestro Proyecto Social (2015), SENA (2014) ofrecen propuestas itinerantes tecnológicas que promueven la inclusión digital mediante la tecnología de la información y comunicación como herramienta para mejorar la calidad educativa. El internet y la capacitación se convierten en centros de estas 
propuestas, así como dotar de los recursos físicos e infraestructura a poblaciones rurales y vulnerables para que se beneficien de este servicio y mejore su calidad de vida.

Muestra un camión itinerante que funciona como Museo Móvil y recorre las periferias del Distrito Federal para dar a conocer al público infantil la obra del pintor mexicano Rufino Tamayo; se desarrollan talleres artísticos que giran en torno a temas como el cosmos, los animales y las frutas. En ellos, crean máscaras de animales, pinturas de agua, ofrendas de frutas para su comunidad, escuela, amigos o familia.

Barroso (2012), muestra Loucultura, una propuesta educativa que describe la experiencia de un grupo de Teatro Universitario Itinerante, basado en las representaciones de situaciones o problemas, integra la motivación y las emociones en el desarrollo de acciones para promover la salud.

Teniendo en cuenta estas experiencias itinerantes en el mundo, nace en Colombia Aula Móvil estrategia de educación no formal e itinerante que recorre las diferentes comunas y corregimientos del Municipio de Soacha llevando actividades lúdico-pedagógicas a los ninos, niñas y sus familias participantes todos los sábados. Estas actividades son implementadas por los docentes en formación a través de la organización de diferentes ambientes de aprendizaje que pretenden potenciar el desarrollo integral de estos niños a través de la pedagogía y el juego como también formar a los padres de familia o cuidadores a través de talleres.

\section{Materiales y métodos}

La investigación se desarrolló mediante el enfoque cualitativo y diseño descriptivo que permite analizar cómo es el proceso educativo itinerante realizado en Aula Móvil como sitio de práctica profesional visto desde los docentes en formación y sus tutores, desde su sentir y opinión. Los instrumentos que se utilizaron para recoger los datos fueron la 35 entrevista semi-estructurada de los docentes en formación y sus tutores, como también la observación participante. 
La población con la que se desarrolló el proyecto en los periodos 2018-1 y 2018-2 fueron: 70 docentes en formación y 1663 niños y niñas. La muestra que se tomó fueron 35 docentes en formación que realizaban su práctica profesional en esos periodos, los cuales se seleccionaron por estar presentes durante todo el periodo de la investigación. Asimismo, 100 niños, niñas y 20 padres de familia de 3 barrios (Ducales, Olivos III y Ciudad de Quito) y 2 veredas (San Jorge y El Charquito) que participaron en el proyecto.

El proyecto se desarrolló teniendo en cuenta tres fases: En la primera, se realizó un diagnóstico del funcionamiento de Aula Móvil. En la segunda, la implementación de las propuestas educativas de los docentes en formación en los diferentes ambientes de aprendizaje. Y la tercera, se analizó como fue ese proceso educativo no formal e itinerante y que elementos lo constituían.

Los datos fueron analizados de forma interpretativa a través de la herramienta Atlas ti y con matrices de vaciado de la información. El análisis giró en torno a seis preguntas orientadoras que permitieron comprender mejor el fenómeno de estudio, y tuvieron en cuenta los siguientes elementos: contexto socio-económico, población participante, y finalidad. Las preguntas de investigación alrededor de esta aula itinerante fueron:

$\S$ ¿Qué es una propuesta educativa?

$\S$ ¿Qué fundamentos epistemológicos se tienen en cuenta en la elaboración de una propuesta educativa para las infancias?

$\S$ ¿Cuáles son los elementos de una propuesta educativa en cuanto al proceso de enseñanza-aprendizaje?

$\S$ ¿En el desarrollo del proceso metodológico de la propuesta educativa tiene en cuenta algún modelo pedagógico y referentes teóricos?

$\S$ ¿Qué tiene en cuenta para elaborar una planeación? ¿La planeación es importante en el trabajo de Aula Móvil? 
$\S$ ¿Qué aspectos tiene en cuenta para que esa propuesta sea pertinente, y realizada con responsabilidad social como profesional de la educación?

\section{Resultados}

Propuesta Educativa itinerante

Una propuesta educativa es el medio que permite transformar o atender una problemática evidenciada en el contexto desde el quehacer como profesionales de la educación. Mediante la articulación de la teoría, práctica e investigación, se identifica y reflexiona sobre esa necesidad, y a partir de ésta, se diseña e implementa un plan de acción que la minimice o solucione.

El proceso de enseñanza-aprendizaje que se implementó en este proyecto de educación no formal e itinerante permite al docente reflexionar sobre las forma idónea en que las niñas, niños y padres adquieran aprendizajes-habilidades (modelo, recursos, espacios, actividades, estímulos) y al mismo tiempo, conoce las características de los participantes (edad, nivel de maduración, características socio-culturales, conocimientos previos) para que esas experiencias significativas sean interiorizadas y les sirvan para la vida.

En el desarrollo de las propuestas se hace necesario contar con material didáctico ofrecido por la universidad con condiciones de estética y calidad. Del mismo modo, el material que es elaborado por las estudiantes no cumple con las condiciones exigidas de estética, durabilidad (transporte) y calidad necesaria para la atención de las infancias en los diferentes barrios y veredas.

Fundamentos epistemológicos de la propuesta no formal e itinerante

El diseño de propuestas educativas para las infancias requiere del manejo e interiorización de conocimientos disciplinares en Educación infantil. Conocer cómo se da la praxeología, qué elementos se tienen en cuenta en el proceso de enseñanza aprendizaje, qué es un ambiente de aprendizaje, didáctica, metodología y lúdica. Todo esto con el propósito de tener claridad al diseñar e implementar las diferentes propuestas educativas en los 
ambientes de aprendizaje. Uno de los problemas más evidentes en el proyecto de investigación es la falta de apropiación de la fundamentación epistemológica, lo que dificulta el diseño y la construcción de las propuestas, la claridad en el objetivo, la construcción de material didáctico y la evaluación de éstas.

Elementos pedagógicos de la propuesta educativa no formal e itinerante

Ambientes de aprendizaje

Espacios que cuentan con recursos físicos, didácticos e interacciones entre los participantes que facilitan la adquisición de competencias cognitivas y sociales importantes para el desarrollo integral de los niños y niñas. Utiliza estímulos y experiencias significativas motivantes para ellos. Los estudiantes en edad infantil necesitan de estímulos visuales, auditivos, corporales, estéticos y de trabajo colaborativo para incorporar nuevos aprendizajes significativo para la vida. La educación no formal e itinerante forma individuos alegres, seguros, propositivos con esperanza, con capacidad de trabajo colaborativo (Molins, 2014). De igual manera, las niñas y niños requieren ambientes agradables y retadores, que propicien nuevos saberes y logren aprendizajes significativos que aporten al desarrollo de las dimensiones humanas (Manrique, 2013).

Algunos ambientes de aprendizaje necesitan ser mejorados, su disposición y uso de material son pobres, no cumplen con los parámetros para la construcción de estos como: que cuente con material didáctico de calidad y pertinencia para el tema trabajado, que en el desarrollo de la actividad propenda por la interacción con otros y el trabajo colaborativo.

\section{Material didáctico}

El uso del material didáctico facilita la adquisición de habilidades y aprendizajes, es el soporte dinámico de este proceso, capta el interés, fortalece la concentración, posibilita la construcción de conceptos de manera autónoma y divierte, de igual modo permite el aprendizaje. Los materiales utilizados por los docentes en formación son de baja calidad, no cumplen con la estética requerida, la durabilidad 
necesaria para el transporte y trabajo con los niños en edad infantil, y carecen de creatividad.

\section{Planeaciones}

La planeación permite organizar la práctica educativa para favorecer el logro de aprendizajes y alcanzar los propósitos planteados en el proceso educativo (Malagón y Montes, 2013). Es la disposición escrita de los temas, recursos y espacios necesarios para implementar las propuestas educativas, teniendo en cuenta el objetivo que se quiera lograr, la metodología que se va a implementar y la forma como se va a evaluar, en conclusión, tiene en cuenta qué, cómo, con qué, cuándo enseñar y cómo y cuándo evaluar. En las planeaciones se evidencia falta de idoneidad en el planteamiento de objetivos, el modelo pedagógico, las características de los niños y niñas participantes, la forma de evaluar, se olvida tener en cuenta el contexto para realizar las planeaciones.

Evaluación

Reflexión argumentativa del proceso de aprendizaje realizado, teniendo en cuenta el contexto, las características de la población, los objetivos planteados, los recursos utilizados, y la metodología implementada. Es un proceso de reflexión continuo para optimizar los procesos de aprendizaje que se dan y puede ser de forma cualitativa o cuantitativa. Permite conocer los logros de los y las estudiantes, como del proceso realizado, si es adecuada la metodología y sí el aprendizaje construido es significativo y con pertinencia social (Ministerio del Poder Popular, 2008). Poco se trabaja la evaluación en el desarrollo de cada actividad, se ejecuta el inicio y desarrollo de la actividad, pero no se concluye con la evaluación. Si se realiza una evaluación general, de cada sesión o encuentro de Aula Móvil, reflexionando sobre los aspectos positivos y negativos que ocurrieron en el encuentro.

Metodología de la propuesta

La metodología es el soporte conceptual con el que se implementa el proceso educativo, responde a la pregunta cómo se va a enseñar. Cuenta con modelos, estrategias y recursos didácticos que facilitan 
la enseñanza y el aprendizaje. En la enseñanza se utiliza un modelo pedagógico (constructivismo, conceptual, dialogante, tradicional, conductista) que orienta la adquisición de conocimientos y el desarrollo de habilidades. De igual manera, se elige la estrategia por medio de la cual se van a trabajar, éstas pueden ser el juego, el arte, los retos, las preguntas problema, el trabajo colaborativo, proyectos de aulas, etc. Según sea la estrategia escogida se establecen los recursos didácticos necesarios para potenciar un proceso dinámico. Las falencias de apropiación de los fundamentos epistemológicos de la educación y educación inicial dificultan la metodología y el desarrollo óptimo de las diferentes actividades y ambientes de aprendizaje.

Pertinencia y responsabilidad social de la propuesta educativa

La propuesta educativa es pertinente cuando parte de las necesidades del contexto que se pretende impactar, apunta a mitigar las necesidades de las comunidades para lograr una transformación social. De igual manera, cuando atiende a las particularidades de la población que será atendida (edad, grado de escolaridad, intereses, gustos, conformación familiar, cultura). El docente tiene un compromiso profesional y social en reconocer los saberes de la comunidad, trabajar con ellos para co-construir proyectos innovadores que atiendan sus necesidades con actitud responsable y comprometida en búsqueda de la calidad de vida justa y en paz. Algunos docentes en formación asisten al Aula Móvil con el propósito de cumplir con su práctica profesional, no tienen compromiso social y tampoco responsabilidad de ofrecer actividades de calidad y pertinentes según el contexto.

\section{Discusión}

La articulación entre la docencia y la investigación permite reconocer las problemáticas de las diferentes comunidades y proponer alternativas de solución o transformación a éstas, con propuestas educativas innovadoras que salgan de la escuela y los atienda en lugares cerca de su vivienda, con condiciones de calidad y pertinencia. Estas propuestas se deben adaptar a los contextos y las necesidades presentadas, en atención a poblaciones vulnerables, de 
las periferias o zonas de difícil acceso en diferentes sitios como parques, salones comunales o zonas verdes.

Los procesos educativos no formales e itinerantes poseen los mismos elementos pedagógicos que la educación formal, como una propuesta pedagógica que explicita los fundamentos epistemológicos en los que se basa, los elementos del proceso de enseñanza-aprendizaje que emplea, el proceso metodológico para su implementación y una planeación con el qué, cómo, para qué y el cómo lo evaluara. Todo esto atendiendo a una problemática específica evidenciada, en un contexto determinado y con una población concreta para que sea pertinente y acertada.

Uno de los principales retos de la educación es ofrecer cobertura total $\mathrm{y}$ de calidad a toda la población, educación formal (colegios y cupos para toda la población escolar) y no formal (curos y espacios de música, danzas, teatro, artes plásticas y literatura) que ejercite las dimensiones del ser para el desarrollo integral de las infancias garantizando condiciones de inclusión, igualdad y equidad para todos los niños y niñas de todas las comunidades.

Es necesario diseñar adaptaciones pedagógicas en este proceso educativo no formal e itinerante, ya que a los encuentros llega población diversa con limitaciones cognitivas, corporales y de comunicación a quienes se les debe garantizar la participación en estos ambientes de aprendizaje y el acceso al material utilizado por todos.

\section{Conclusiones}

En zonas periféricas y rurales el gobierno debe garantizar el acceso a esta educación no formal e itinerante para disminuir problemáticas como la delincuencia, drogadicción, comportamientos violentos y depresiones o la desesperanza de falta de oportunidades.

La responsabilidad social de la educación superior en la atención de las problemáticas de la comunidad es vital para la transformación de los diferentes contextos y alcance de sociedades más justas y equitativas. En este sentido, los docentes tienen la responsabilidad de diseñar e implementar propuestas educativas en la atención de las 
infancias y sus familias que promuevan el mejoramiento de la calidad de vida, una sociedad más incluyente y democrática (SENA, 2014).

En zonas periféricas y rurales el gobierno debe garantizar el acceso a esta educación no formal e itinerante para disminuir problemáticas como la delincuencia, drogadicción, violencia y depresiones o la desesperanza de falta de oportunidades.

\section{Referencias}

Barroso, S.; Lappan, N.; Simeone de Almeida, M. (2012). Teatro Universitario en la Educación como una estrategia para la Salud Mental. Revista Ciencia em Extensáo. 8(3), 118-127. Recuperado de:

https://ojs.unesp.br/index.php/revista_proex/article/view/693/748

Bernal, A. H. (2012). Radio Sutatenza: un modelo colombiano de industria cultural y educativa. Boletín Cultural y Bibliografico, Banco de la República, 46(82), 4-41. Recuperado de: https://publicaciones.banrepcultural.org/index.php/boletin_cultural/ article/view/67

Covarrubias, K.; Hernández, A.; González, C. (2016). Análisis etnográfico sobre el impacto social y cultural del Microteatro itinerante en grupos vulnerables en cuatro municipios del estado de Colima, México. Revista Estudios sobre las Culturas Contemporánea. Época III. 22(449, 99-126.

Dominguez, J.; Rama, C. (2012). La Responsabilidad Social Universitaria en la Educación a Distancia. Chimbote, Perú: Universidad Católica Los Ángeles de Chimbote.

Granada, R. C.J. (2012). La Responsabilidad Social Universitaria en la Educación a Distancia. Perú: Universidad Católica de los Ángeles Chimbote

Malagón y Montes, G. (2013). Las competencias y los métodos didácticos en el jardin de niños. México. Editorial Trillas. 
Manrique, A.; Gallego, A. (2013). El material didáctico para la construcción de aprendizajes significativos. Revista Colombiana de Ciencias Sociales, 4 (1) ,101-108. Recuperado de: file://C:/Users/User/Downloads/DialnetElMaterialDidacticoParaLaConstruccionDeAprendizaje5123813.pdf

Ministerio de Educación Nacional. (1994). Ley General de Educación. Recuperado de: https://www.mineducacion.gov.co/1621/articles85906_archivo_pdf.pdf

Ministerio de Educación Nacional. (2004). Altablero, El periódico de un país que educa y que se educa. Recuperado de https:/www.mineducacion.gov.co/1621/article-87375.html

Ministerio del Poder Popular para la Educación. (2008). La evaluación en el sistema Educativo Bolivariano. Educere: La revista Venezolana de Educación. Educere, 12(40), 196-206. Recuperado de: https://www.redalyc.org/pdf/356/35604024.pdf

Molins, M., Cano, E., Lorenzo, N. (2014). Maria Montessori: el Método de la Pedagogía Científica en El legado pedagógico del siglo XX para la escuela del siglo XXI. Barcelona: Alfaomega.

Nuestro Proyecto Social Educativo, B. (2015). Biblioburro. Obtenido de http://biblioburrosinfronteras.blogspot.com/

Rodríguez-Gutiérrez, F. (2017). La Universidad Itinerante de la Mar, una Plataforma para el conocimiento Geográfico del Mundo Actual. Revista de Estudios Andaluces (REA), 34(1), 255-292. Recuperado de:

https://idus.us.es/xmlui/bitstream/handle/11441/66301/Art9.pdf?se quence $=1 \&$ isAllowed $=\mathrm{y}$

Secretaria de Educación Distrital. (2014). Ambientes de aprendizaje. S.D. Educación, Ambientes de aprendizaje para el desarrollo humano. Reorganización por ciclos. Herramienta de consulta y orientación para el diseño. Bogotá, Colombia: Fundación de Pedagogia Conceptual Alberto Merani. 
SENA. (2014). Aulas Móviles SENA. Servicio Nacional de Aprendizaje SENA. Colombia. Recuperado de: http://www.sena.edu.co/esco/formacion/Paginas/aulasMoviles.aspx

Torres, F. (2016). Aulas itinerantes en el circo: de la escuela de ladrillo y cemento a la escuela viajera sobre ruedas. Revista de Educación Inclusiva, 9(3), 63-74 .Recuperado de: https://www.revistaeducacioninclusiva.es/index.php/REI/article/vie $\mathrm{w} / 253 / 247$

UNAD. (2018). Maestro Itinerante. Bogotá D.C, Colombia: UNAD. Recuperado de: https://gaf.unad.edu.co/proyectos/maestroitinerante 